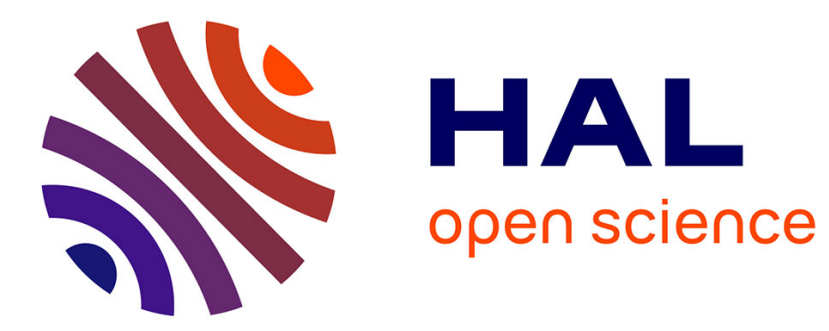

\title{
A Semiotic Approach for Guiding the Visualizing of Time and Space in Enterprise Models
}

\author{
John Krogstie, Alexander Nossum
}

\section{To cite this version:}

John Krogstie, Alexander Nossum. A Semiotic Approach for Guiding the Visualizing of Time and Space in Enterprise Models. 15th International Conference on Informatics and Semiotics in Organisations (ICISO), May 2014, Shanghai, China. pp.74-86, 10.1007/978-3-642-55355-4_8 . hal-01350912

\section{HAL Id: hal-01350912 \\ https://hal.inria.fr/hal-01350912}

Submitted on 2 Aug 2016

HAL is a multi-disciplinary open access archive for the deposit and dissemination of scientific research documents, whether they are published or not. The documents may come from teaching and research institutions in France or abroad, or from public or private research centers.
L'archive ouverte pluridisciplinaire HAL, est destinée au dépôt et à la diffusion de documents scientifiques de niveau recherche, publiés ou non, émanant des établissements d'enseignement et de recherche français ou étrangers, des laboratoires publics ou privés. 


\title{
A Semiotic Approach for Guiding the Visualizing of Time and Space in Enterprise Models
}

\author{
John Krogstie ${ }^{1}$, Alexander Nossum ${ }^{2}$ \\ Norwegian University of Science and Technology (NTNU) ${ }^{1}$, Norkart A/S ${ }^{2}$ \\ krogstie@idi.ntnu.no
}

\begin{abstract}
Even if geographical aspects such as location are included already in the Zachman framework (as the where-perspective), it is not common to have detailed geographical aspects included in enterprise models. Cartography is the science of visualizing geographical information in maps. Traditionally the field has not included conceptual relationships that you find in enterprise models. Both cartography and enterprise modelling have developed guidelines for obtaining high quality visualizations. SEQUAL is a quality framework developed for understanding quality of models and modelling languages based on semiotic theory. In cartography such frameworks are not common. An adaptation of SEQUAL in the context of cartographic maps called MAPQUAL has been presented earlier. Differences between quality of maps and quality of conceptual models, pointing to guidelines for combined representations have been performed, and we try in this paper to investigate the utility of these guidelines in a simple trial. The result of the trial is presented, indicating that it is possible to represent conceptual, temporal, and spatial aspects in the same models in many ways, but that the choice of main perspective should depend on participant appropriateness.
\end{abstract}

Keywords: Quality of models, Spatial enterprise models, Maps, Semiotics

\section{Introduction}

A conceptual model is traditionally defined as a description of the phenomena in a domain at some level of abstraction, which is expressed in a semi-formal or formal visual language. An enterprise model can be regarded as a kind of conceptual model. The field has spawn from information systems development and computer science with methodologies like Data Flow Diagram (DFD), Entity Relationship diagrams (ER) and more recently Unified Modeling Language (UML), Business Process Model and Notation (BPMN) and Archimate. The languages used for conceptual modelling largely contain nodes and links between nodes, and containment relationships. In enterprise modelling a number of perspectives to modelling are distinguished. For instance the Zachman Framework [22] describes 6 perspectives; What (material) it is made of, How (process) it works and Where (location) the components are, relative to one another, Who is involved, When are tasks done relative to each other and Why. In conceptual and enterprise modelling, we traditionally deal with what (data 
modelling), how (process modelling), who (organizational and actor modelling), when (behavioural and temporal modelling), and why (goal-oriented modelling). On the other hand the location aspect (where) is seldom dealt with in detail, although as we see e.g. in the development of BIM [21] of buildings that topological aspects get more and more important to represent.

Cartography on the other hand, focuses on aspects of location through maps. Maps at first sight appear to be very different from conceptual models. However, many similarities among these representations can be found.

The ultimate goal of the work is to develop an understanding of quality of enterprise models when also including geographical/topological constructs. To get to this we have earlier developed a framework for understanding and assessing quality of maps (MAPQUAL [18]), based on the SEQUAL-framework [10] for quality of models and modelling languages. Differences between SEQUAL and MAPQUAL are used to assess how combined geographical and conceptual models should be developed to achieve high quality models, and the aim of this paper is to report in experiences with modelling notations that takes into account both conceptual, topological and temporal issues.

In section 2, we present background on quality of maps and conceptual models. Section 3 describes the case and section 4 present a simple trial with two alternative visualizations of situations in the case-domain. In section 5 we sum up the experience and describe further work on an integrated approach.

\section{Background and Related Work}

A map is a kind of model. An underlying assumption has been that cartographic maps represent, primarily, geographic concepts. Some research has been put into applying cartographic visualization techniques as described by [4] on general non-geographic information $[1,15,20]$ and the opposite, applying general information visualization techniques on geographic information. However, little work has looked on combining conceptual models with cartographic maps.

Earlier work on quality of models and quality of maps described in MAPQUAL [18] has illustrated that the main semiotic levels related to quality of models as described in SEQUAL applies also to maps. There are also distinct differences, which is a challenge when you want to visualize both geographical and conceptual aspects.

The differentiation between language and model (map) are usually not discussed in cartography. There exists no tradition of defining the syntax for languages for making maps, although standardizations towards both symbol sets and rules for applying them exist. MAPQUAL recognize this and aims at investigating how existing cartographic research can be structured following the SEQUAL structure inspired by semiotics.

Generally the visualization in maps can be said to comprise three graphic primitives; point, line and area and relations between these (Points being within an area, line crossing an area or being the border of an area etc). This is inherently different from meta-meta models in conceptual modelling which usually comprise of only nodes and links between nodes, in addition to visual containment. 
The main aspects of the basic MAPQUAL [18], extended to take into account additional aspects of indoor maps [16] is described here.

\subsection{Comparing Quality of Maps and Quality of Models}

We here discuss the different levels of quality according to the semiotic ladder first described in the work of Stamper [6].

- $\quad$ Physical quality: The basic quality goal is that the model exists physically and is available to the relevant actors. Cartography is traditionally more geared towards making tangible representation of maps (i.e. printed maps) -although this is shifting towards more intangible representations for instance in a software environment (i.e. web mapping tools). SEQUAL focus much on guidelines for a modelling environment and different functionalities that it should provide. It should be noted that these guidelines are adapted to an information systems context, however, the guidelines should hold true for a cartographic environment as well. Also aspects of security (privacy) and currency as discussed in [16] is relevant for both types of representations.

- $\quad$ Empirical quality deals with comprehension when a visual model $\boldsymbol{M}$ is read by different social actors. MAPQUAL is significantly different from SEQUAL in this area. This is mainly due to the differences in meta-meta model discussed above. Colours are heavily used in cartography to separate different concepts from each other. In conceptual modelling the use of colours has been avoided to a large degree. Gopalakrishnan et al. [8] suggest using colours more in conceptual models. The inherent topological attributes of cartographic concepts often restricts the freedom of layout modifications, such as choosing where a concept should be placed on a map. Guidelines for increasing empirical quality of conceptual models base themselves, mostly, on the freedom of layout, supported by guidelines for graph aesthetics. These guidelines are thus not directly applicable to a map. In cartography one could see the aesthetics and geographical attributes as orthogonal dimensions. Conceptual modelling and cartography share the background for the guidelines for empirical quality.

- Syntactical quality: Is the model according to the syntax of the language. In cartography there is a lack of definition of formal languages in designing maps.

- Semantic and perceived semantic quality is the relation between the domain, map/model and social actor knowledge and interpretation. Thus, this facet is assumed to be generally applicable for cartography as well as for conceptual modelling. In cartography the quality of the data, in terms of measure errors, is quite common to use as a semantic quality measure.

- Pragmatic quality relates to that human interpreters understand the model. MAPQUAL does not include an extensive investigation in the research of human interpretation of maps in cartography, but recognise that there are many similarities between this and SEQUAL' focus on human interpretation of models. MAPQUAL and SEQUAL are thus more or less equal with respect to pragmatic quality, although with increasing focus on interactivity of digital maps [16]. 
- Social quality: Do different stakeholders agree on their interpretation of the model. MAPQUAL base the discussion of social quality of cartographic maps solely on the discussion of social quality in SEQUAL.

- Deontic quality: Do the model help to achieve the overall goal of modelling? Emphasise is put on how maps can support the achievement of goals of the map.

Looking on language quality we have found the following:

- Domain appropriateness: Due to the lack of discussion and formal separation of domain and language in cartography, MAPQUAL is similar to SEQUAL with respect to domain appropriateness (is it possible to represent the domain using the language).

- Participant appropriateness: Cartography has a tradition of exploiting the "natural" or cognitive knowledge of participants to a large extent. E.g. the use of colour for type of areas in a map reflects the colour in the real world. In conceptual modelling the tradition of creating a new language and thus disseminate this knowledge in a tailored way is more common.

- Modeller (cartographer) appropriateness: Similar to participant appropriateness, MAPQUAL and SEQUAL are similar with respect to modeller appropriateness.

- Comprehensibility appropriateness: Comprehensibility is divided into two areas; conceptual basis and external representation. Conceptual basis comprise the discussion on which concepts that are included in the language. SEQUAL provides several concrete guidelines for the conceptual basis. These guidelines have validity in cartography as well as for conceptual modelling. External representation focus on how the notation of the language is formed, i.e. the graphical aspects of the language. In this area there are significant differences between MAPQUAL and SEQUAL. Cartography has a strong tradition of investigating graphic design principles based on so-called visual variables [4]. Traditionally maps have a larger focus on the use of colours and the use of texture as a visual technique. SEQUAL encourage being able to support a free approach to composition of symbols. Such free composition of symbols cannot be a general guideline in cartography since the geographical reality often is constraining this freedom.

- Tool appropriateness: Tool appropriateness is traditionally not considered in cartography. Thus MAPQUAL adopts SEQUAL on the discussion of tool appropriateness, although with less focus on executional semantics.

- $\quad$ Organisational appropriateness: Here MAPQUAL focus more on a cartographic context and the current standardisation efforts in this area.

Thus, two interrelated facets have large differences: comprehensibility appropriateness for language quality and empirical quality for map/model quality. When investigating and comparing MAPQUAL and SEQUAL, we devise some important guidelines when using both conceptual and geographical aspects:

1. Clearly discriminate between geographical oriented lines and conceptual lines.

2. Clearly differentiate between nodes (concept) which are often depicted by a geometric shape, and geographic areas (by texture or colour for instance) 
3. Indicate topological information by positioning of conceptual nodes according to the topology when possible.

4. Position concepts according to their temporal nearness.

5. Use visual variables where appropriate, especially the use of colour and shading for differentiation is necessary for integrated models.

6. Design the visualization based on the participants' cognitive metaphor of the most important information attribute. For instance, temporal attributes tend to be lean towards a sequential metaphor. Spatial attributes, like nearness, tend to lean towards a distance metaphor (i.e. closer is nearer).

\section{Quality of Integrated Conceptual and Topological Models}

In the investigation of developing quality guidelines for integrated visualizations that exhibit both conceptual and geographic information, a case study has been performed. The case study is in the health-care domain, which is an area where space support is found to be very important [9] and which lends itself well to these kinds of visualizations. Research suggests that providing awareness of the hospital environment is one mean to lower the complexity of the decision-making. Both a focus towards the spatial dimension (i.e. location), but also the conceptual dimension (i.e. state, relationship etc.) is needed $[2,3]$.

The spatial dimension in indoor environments is commonly visualized either directly in a floor-plan (i.e. an indoor map) [13] or as an attribute in a diagram-like fashion [3]. Both approaches aim at visualizing the spatial dimension as well as the conceptual dimension including relationships, states and similar. However, both approaches focus the visualization towards their respective field (i.e. floor map on the spatial dimension, diagrams on the conceptual dimension) without successfully obtaining a good communication of both dimensions at the same time. This section will describe the background for the case and the perspective chosen for the trial, as well as a concrete situation that instantiates the information space under investigation.

\subsection{Self-coordination of Hospital Staff}

The primary focus for the case is the self-coordination of hospital-staff. A typical work situation for hospital staff includes various tasks that can either be scheduled or occur spontaneously. The tasks may require different equipment, several other staff members may be involved in a task, either throughout the whole duration of the task or only briefly, and often involving patients. Additionally, the staff member may have special interests in specific patients. This can be modelled by concepts in the information space, with relationships among them. Moreover, all the concepts have a spatial relationship of some sort. Concepts also have several non-physical properties, such as state, importance, staff type and so on.

The spatial dimension of the information is regarded as highly important and relevant for collaboration and coordination. The absolute location of concepts is not necessarily what best satisfies the actors' information needs. Deduced information, 
based on the absolute location of concepts may prove to be more suitable, especially towards the topology of concepts. Topology aspects may be spatial or temporal. Spatial topology is commonly exhibited in cartography for instance in subway maps, where less emphasis is put on distance than in topographical maps. Temporal aspects can be deduced by, for instance, the travelling distance divided by the travelling speed. In an indoor environment, this is very useful as the distances may be large, but conceptual entities can travel fast, for instance by elevators across floors. Additional obstacles that slow actors down are also present, such as wardrobes, bathrooms, disinfection areas, etc, where actors are held up temporarily.

\subsection{Concrete Case Relative to Trial}

Since the conceptual information model can attain fairly high complexity, it is important to constrain the case-study to a moderately complex sub-domain. The casestudy revolves around one specific actor as the intended main user. The user has certain interests, or relevant information associated with him/her, this information is a subset of the total information available. The goal for the trial was to provide a visual communication method which satisfies this users specific information needs. However, the method developed and investigated should also be able to be adapted to satisfy similar scenarios with other user types.

The scenario focuses on a typical working day for an anaesthetist. An anaesthetist's tasks are typically distributed throughout the hospital and each task has a small time span. Often the anaesthetist is working in parallel on two or more tasks, providing a need to self-coordinate in an effective matter, often requiring the knowledge of spatial attributes.

Development of the visual representations could be performed in several different ways, each with different benefits. For the initial investigation, a paper-prototype inspired approach was taken. Paper-prototypes supports for fairly rapid development, allowing for several ideas to be manifested and iteratively developed. The development of paper-prototypes ended with primarily two different kinds of visual representation methods, spatial Gantt charts (see example in Fig. 2) and Spatial Circles (see example in Figure 1). Both take into account the guidelines in the end of section 2, but Spatial Gantt chart have a higher emphasis on the temporal dimension. Due to the previous experiences of the subject (with Gantt-charts), we expected that the Spatial Gantt chart would perform best. A full description of the notation and snapshots used in the trial is found in [17].

\section{Trial}

The design of the trial was inspired by experiments conducted by [19] on differences between two conceptual modelling techniques. It should, however, be noted that the trial in this project was never intended to be as comprehensive as Opdahl and Sindre [18], especially with respect to the number of participants. Since it was not 
meaningful to use proxies (e.g. students) in the case, the number of available subjects was also too small to use statistical methods.

The trial is designed with strong inspiration from both the Latin-Square experimental design method, work by [7] and the Technology Acceptance Model (TAM) [5]. The main intention of the trial is to investigate the properties of comprehension of the different types of visual representations and their acceptance.

In the trial the participants were given an introduction to the area, and then presented with a tutorial of the visualization to be presented. Then the visualization representing the scenario snapshot was presented. Instructions were given to answer a questionnaire -the time frame of this was 5 minutes. This process was performed iteratively for each of the snapshots in the scenario, but by changing the order of visualization types presented i.e. changing the order in which the different visualizations were presented, and having different visualizations representing similar information for the two different trials to leverage the potential learning factor for the visualization types. Additionally it is desirable to be able to compare the two different visualization types against the same information without repeating the presentation of the same scenario snapshots to the same participant. One potential shortcoming of this design is the differences in the participants -if the number of participants is small the impact of this will increase and can lead to wrong conclusions if not taken into account.

The questionnaires are essentially of two different types, one which is to be answered during the presentations of the different visualizations and one to be answered after finishing the presentations. One questionnaire for each of the scenario snapshots was created. The users' comprehension and potential knowledge gain from the visualization are evaluated by investigating the questionnaires. Comprehensibility deals with whether the user understands what the visualization depicts. Knowledge gain deals with whether the user is able to use the information in the visualization and potentially create new knowledge, or take decisions based on the information gained. Typical questions included for these two topics are:

- Comprehensibility "Is task 1 far away?, When is task 2 starting?

- Knowledge gains "What do you decide to do next?

The post-presentation questionnaire was more comprehensive and aims primarily at investigating the user acceptance of the two different visualization types. Categorization and questions were inspired by the Technology Acceptance Model and [19] with the following categories:

- $\quad$ Perceived usefulness

- Perceived ease-of-use

- Intention to use

The post-presentation questionnaire used a 5-point Likert scale with the "opposing statements" format of the questions. The answer options indicated to which degree the subject agreed with the statements. In order to avoid repetition and monotony, the questions ordering was randomized. 


\subsection{Results from the Trial}

Two participants were recruited to the trial, both staff at the local hospital. One participant was an anaesthetist and the other a general practitioner. Neither one received any compensation for their participation. Both trials were set up identically with respect to both equipment and introduction of the participants to the trial. Each trial took approximately 30 minutes.

This section will present and discuss the results from the trial categorized according to comprehensibility, knowledge gain and technology acceptance briefly (due to the strict page limitations of the conference).

Comprehensibility: The results of the comprehensibility investigation were calculated as an average over all questions related to comprehensibility, regardless of the information depicted. The questions were not designed to necessarily have a yes/no answer. Coding of the answers given was performed by mapping each answer to correct, semi-correct or wrong. This could potentially introduce bias or errors in the results since it was done by one person only, however due to the limited scope of the trial this was regarded as tolerable. The results indicate a slightly higher proportion of correct answers for the Spatial Circle layout than the Spatial Gantt layout. However the difference is marginal.

Knowledge gain: The results were calculated by first coding the answers in three different categories; correct, semi-correct and wrong. It is noted, as for results under comprehensibility, that this can introduce biased results. A standard arithmetical average was then calculated for each of the category over the answers related to knowledge gain. A differentiation between the two visualization types was kept, in order to compare their performance. The results indicate that Spatial Circle layout performs slightly better than the Spatial Gantt layout. However, the difference between the two is marginal.

Technology acceptance: The final and most comprehensive questionnaire revolved around investigating the participants' acceptance of the visualizations. Results from the technology acceptance investigation were produced by calculating an average of the degree of acceptance in the different categories of acceptance for each of the visualizations. Additionally, an average over the three categories was calculated to provide a summary of the acceptance. The degree of acceptance used the 5-point Likert scale which was coded as a range from -2 to +2 . The result was that the Spatial Gantt layout appears to be superior to Spatial Circle layout in all categories of acceptance. Spatial Circle layout received negative degree of acceptance in all categories. It is not justifiable to draw a solid conclusion based on these results however the results is regarded as indicative with respect to the comparison between the two visualization types.

The superior acceptance of Spatial Gantt is attributed to the participants' familiarity with the Gantt, or Gantt-like, metaphor. This familiarity was recognized in the design of the visualization types and was thus not unexpected. On the other hand, it is interesting that even if the performance (as for comprehension and knowledge gain) 
seems relatively equal, the difference in acceptance of the approach appear this clear. Similar results are found in other studies of modelling notations [8].

\section{$5 \quad$ Conclusion and Future Work}

This work is aimed at investigating properties of visual representation of geographical, temporal and conceptual representations. Integrating spatial and nonspatial information is needed in meeting the increased amount of spatial and conceptual information. Information is increasingly including a spatial attribute [11] which indicates a need to understand how integrated visual representations works. One way of eliciting this is to merge current understanding of quality in cartography and conceptual modelling and thus create an integrated understanding of quality. In earlier work, the development of MAPQUAL has contributed to a broader understanding of integrated visual representations.

In order to investigate properties of different visual representation techniques a case study was performed. The health-care domain was chosen, with the specific scenario of an anaesthetist work day. Two distinctly different visual representation methods were developed for the scenario following the guidelines for mixed representations. In order to investigate their appropriateness a simple trial was designed and conducted. Due to limited resources the trial was intended to be illustrative rather than provide statistically valid conclusions, largely due to the limited number of participants involved. Further efforts relating to integrated visual representations can benefit by revising and extending the experiment design based on the experience presented in this article.

Through the investigations of visual representations, several issues were discovered. The information was perceived by the participants as through a sequential metaphor, favouring a sequential alignment of the temporal attributes. Thus, the nonsequential layout (i.e. Spatial Circles) was not favoured for the scenario. More generally this is formulated as a guideline which relates to sequentiality of the visual representation relative to the participants cognitive metaphor of the information.

Introducing spatial attributes to a traditionally non-spatial information space, such as schedule information, is new and unfamiliar to the participants. This can introduce issues with the acceptance of the visual representation developed. Moody [15] identifies a similar issue with development of modelling languages.

The investigations did not look on tool support for any of the visual representations. This is intentionally left up to further work. It is strongly encouraged that further efforts delve into the investigation of relevant techniques for tool support. Filtering techniques from conceptual modelling combined with generalization and zooming techniques from cartography and GISc are reckoned to be highly successful and a necessity for the success of integrated visual representations. Additional tool support could introduce the notion of interactive models/maps.

Experience from this work has illustrated that integrating visual representation techniques from cartography and conceptual modelling is feasible and potentially useful. The work reported here has only briefly investigated some of this potential. The close collaboration with domain experts from the application domain proved 
highly beneficial and led to development which otherwise could not have been possible. Further investigations similar to this one, is encouraged to look into the perceived usefulness for the participants and the effects on collaboration between relevant participants. Additionally, more complex scenarios should be developed and investigated, preferably spatial-intensive scenarios, i.e. where spatial attributes are recognized in advance as important by the participants.

\section{References}

1. van der Aalst, W.: TomTom for Business Process Management Proceedings CAiSE'2009, Amsterdam, June, Springer (2009)

2. Bardram, J., Bossen, C.: Mobility Work: The Spatial Dimension of Collaboration at a Hospital. Computer Supported Cooperative Work 14:131-160 (2005)

3. Bardram, J., Hansen, T.R., Soegaard, M.: AwareMedia - A Shared Interactive Display Supporting Social, Temporal, and Spatial Awareness in Surgery. Proceedings of CSCW'06 November 4-8 Banff, Alberta, Canada. (2006)

4. Bertin, J.Semiology of Graphics: Diagrams, Networks, Maps. Madison, WI: University of Wisconsin Press (1983)

5. Davis, F.: Perceived usefulness, perceived ease of use, and user acceptance of information technology, MIS quarterly pp. 319-340 (1989).

6. Falkenberg ED, Hesse W, Lindgreen P, Nilsson BE, Oei JLH, Rolland C, Stamper RK, Assche FJMV, Verrijn-Stuart AA, Voss K. A Framework of information system concepts - The FRISCO Report, IFIP WG 8.1 Task Group FRISCO (1996)

7. Gemino, A. and Wand, Y.: Evaluating modeling techniques based on models of learning, Communications of the ACM 46(10), 79-84. (2003)

8. Gopalakrishnan, S., Krogstie, J., \& Sindre, G. Capturing Location in Process Models: Comparing Small Adaptations of Mainstream Notation. International Journal of Information System Modeling and Design (IJISMD), 3(3), 24-459 (2012).

9. Gulliver S. R., Grzybek H., Radosavljevic M., Wiafe I. 'Changing Building User Attitude and Organisational Policy towards Sustainable Resource use in healthcare', Health Policy and Technology, 2(2): 75-84. (2013)

10. Krogstie, J. Model-based Development and Evolution of Information Systems Springer (2012)

11. MacEachren, A. and Kraak, M.: Research challenges in geovisualization, Cartography and Geographic Information Science 28(1), 3-12. (2001)

12. Marjamaa, R., Torkki, P., Torkki, M. and Kirvela, O.: Time Accuracy of a Radio Frequency Identification Patient Tracking System for Recording Operating Room Timestamps, Anesthesia \& Analgesia 102(4), 1183. (2006)

13. McCarty, J. F., Meidel, E. S.:ActiveMap: A Visualization Tool for Location Awareness to Support Informal Interactions. In Handheld and Ubiquitous Computing (HUC '99) (1999)

14. Moody, D.: Theoretical and Practical Issues in Evaluating the Quality of Conceptual Models, Data \& Knowledge Engineering 55(3), 243-276 (2005)

15. Moody, D., van Hillergersberg, J.: Evaluating the Visual Syntax of UML: Improving the Cognitive Effectiveness of the UML Family of Diagrams. In: Proceedings of Software Language Engineering (SLE 2008) September 29-30, Toulouse, France (2008)

16. Nossum, A. Developing a Framework for Describing and Comparing Indoor Maps. The Cartographic Journal 50 (3) pp 218-224 (2013)

17. Nossum, A.: Visual Representation of Integrated Information, Master Thesis, Norwegian Technical University of Science and Technology (2009) 
18. Nossum, A. and Krogstie, J.:Integrated Quality of Models and Quality of Maps, Enterprise,14th International Conference, EMMSAD 2009, Held at CAiSE 2009, Amsterdam, the Netherlands, June 8-9, Proceedings, Springer, p. 264. (2009)

19. Opdahl, A. and Sindre, G.: Experimental comparison of attack trees and misuse cases for security threat identification, Information and Software Technology 51(5), 916-932. (2009)

20. Skupin, A., Fabrikant, S.: Spatialisation Methods: A Cartographic Research Agenda for Non-geographic Information Visualization, Cartography and Geographic Information Science 30(2), 99-119 (2003)

21. Succar B.Building information modelling framework: a research and delivery foundation for industry stakeholders. Automation in Construction 2009;18(3):357-75.

22. Zachman, J. A.: A framework for information systems architecture. IBM Systems Journal, 26(3), 276-291 (1987) 


\section{Appendix A: Examples of visualizations}

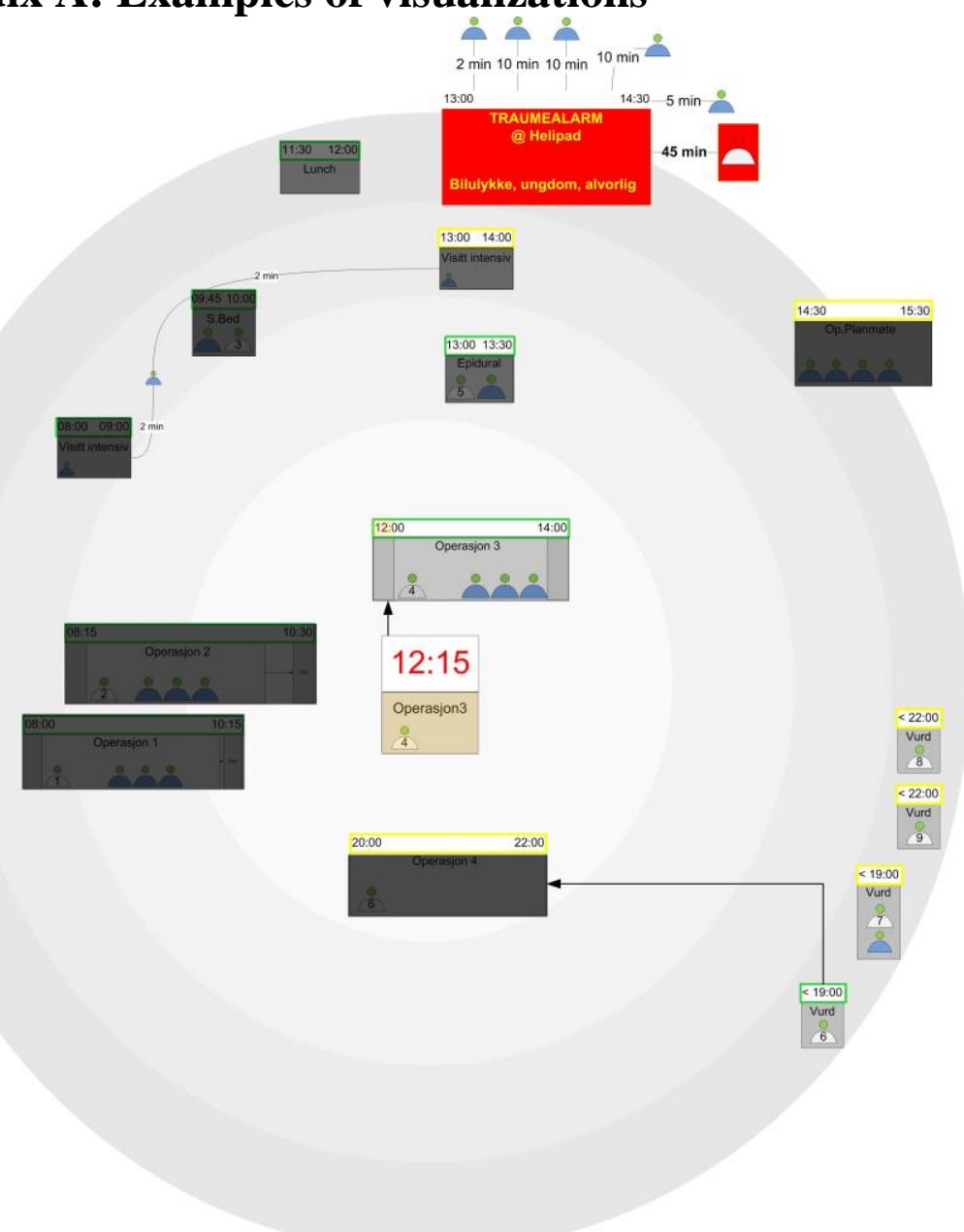

Fig. 1: Example on Spatial Circles 


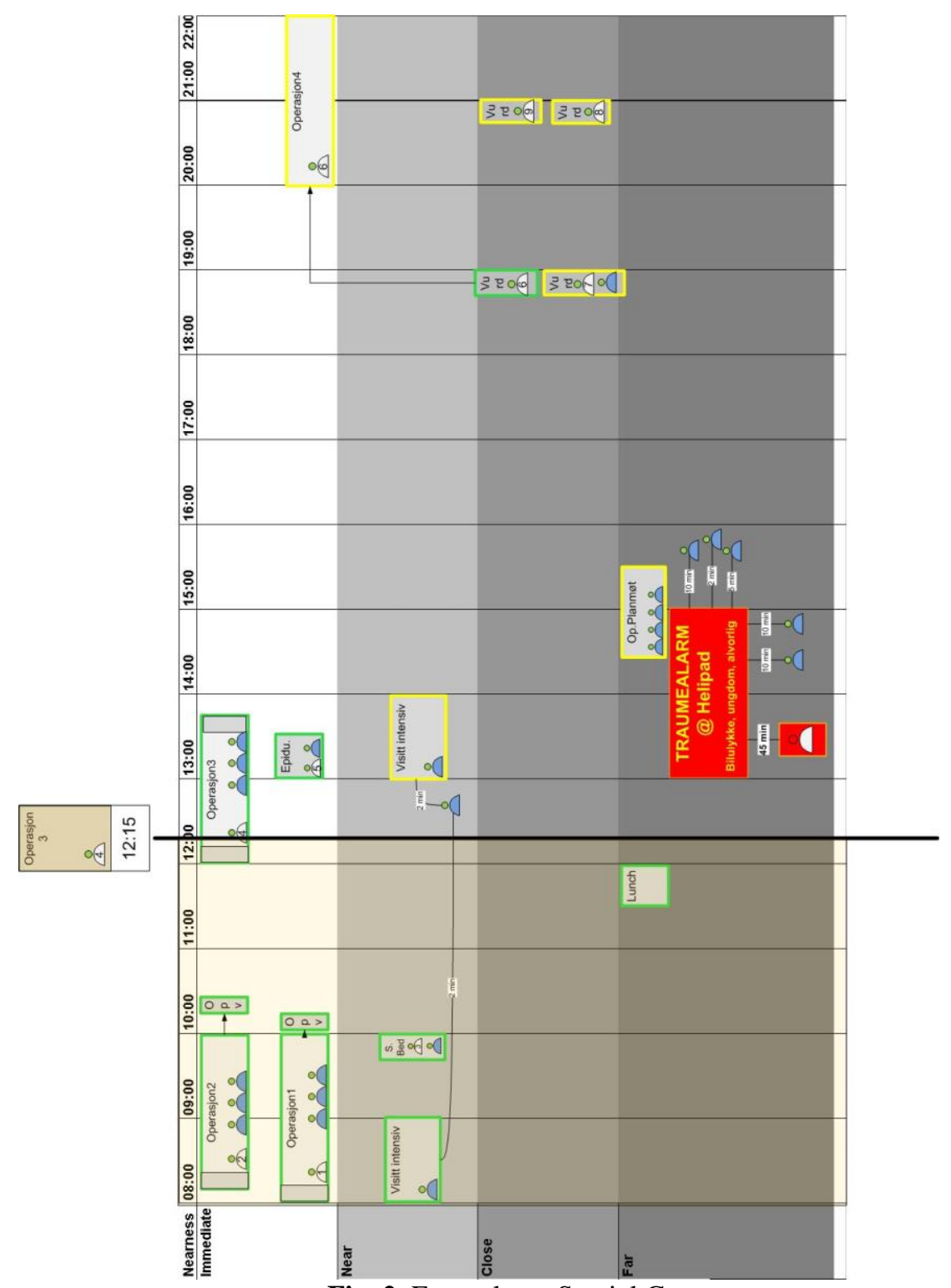

Fig. 2. Example on Spatial Gantt 